\title{
Thanks to the Reviewers
}

The Editors and the members of the editorial board would like to thank the following individuals who have given their time to act as reviewers for the Journal of Vascular Research. Their expertise and effort have been an invaluable help toward the aim of achieving a journal of originality and excellence in the field of vascular science.

M. Adams, Charlottesville, VA R.W. Alexander, Atlanta, GA M. Allen, Seattle, WA B.M. Altura, Brooklyn, NY

A. Arner, Lund

W. Auch-Schwelk, Berlin J. Auwerx, Nice

N.L. Baenziger, St. Louis, MO

J.R. Baker, Birmingham, Alabama

K.M. Baker, Danville, PA

J.B. Bassingthwaighte, Seattle, WA

E. Battegay, Basel

G.L. Baumbach, Iowa City, IA

D.R. Bell, Albany, NY

P.R. Bell, Leicester

R.D. Bevan, Burlington, VT

R.S. Blank, Charlottesville, VA

A. Bobik, Prahran

J. Boyer, Chapel Hill, NC

M. Braide, Gothenburg

J.E. Brayden, Colchester, VT

R. Busse, Freiburg

J. Campbell, Brisbane

W.M. Chilian, College Station, TX G.M. Chisolm, Cleveland, Ohio W.C. Claycomb, New Orleans, LO

A.W. Clowes, Seattle, WA

J.P. Clozel, Basel

M. Clozel, Basel

T. Cocks, Prahran

R.A. Cohen, Boston, MA 


\section{J. Culman, Heidelberg}

A.P. Davenport, Cambridge MJ. Davis, College Station, TX

F. DeClerck, Beerse J.G.R. DeMey, Maastricht S.R. Doctrow, Boston, MA

L. Edvinsson, Malmö

E.O. Feigl, Seattle, WA

J. Folkman, Boston, MA

P. Gasser, Basel

J.F. Giudicelli, Le Kremlin-Bicêtre

T. Godfraind, Bruxelles

P. Gohlke, Heidelberg

M. Goiny, Stockholm

R.W. Gore, Tucson, AZ

A.I. Gotlieb, Toronto

K. Goto, Tsukuba Ibarak

A.A. Gottlieb, New Orleans, LA

A. Gourgos, Boston, MA

T. Griffith, Cardiff

A.W.A. Hahn, Basel H. Haller, Berlin J.M. Hamlyn, Baltimore, MD S.B. Harrap, Victoria P.D. Harris, Louisville, KY D.G. Harrison, Atlanta, GA M. Hasenkam, Aarhus G.T. Haupert, Boston, MA A.M. Heagerty, Manchester D.D. Heistad, Iowa, 10

D. Henrion, Burlington, VT

A.G. Herman, Wilrijk

B. Himpens, Leuven

G.D.S. Hirst, Parkviíle

V.E. Hjortdal, Aarhus

E. Högestätt, Lund

R.L. Hoover, Nashville, TN

J.W. Hopewell, Oxford

0. Hudlicka, Birmingham

\section{L.J. Ignarro, Los Angeles, CA}

M. Jacobs, London

W.F. Jackson, Kalamazoo, MI

P.E. Jensen, Aarhus

R.A. Johns, Charlottesville, VA

H. Karaki, Tokyo

G. Karlstrom, Gothenburg

N.F. Kassell, Charlottesville, VA

N. Korsgaard, Aarhus

R.J. Korthuis, Shreveport, LO

D.M. Larson, Boston, MA

T. Ledet, Aarhus 
R.M.K.W. Lee, Hamilton

A.F. Lever, Glasgow

D.C. Linch, London

J. Linden, Charlottesville, VA

A.L. Loeb, Philadelphia, PA

J. Loscalzo, Boston, MA

GD. Lowe, Glasgow

B. Lowell Langille, Toronto

T.F. Lüscher, Basel

M.W. Majesky, Houston, TX G. Mancia, Milano

T. Masaki, Tsukuba Ibaraki K.I. Maynard, Boston, MA

1. McMurty, Denver, CO

R. Medford, Atlanta, GA

GA. Meininger, College Station, TX K. Messmer, München

461

V.M. Miller, Rochester, MN P. Molenaar, Cambridge

F. Mollinedo, Madrid

E. Monos, Budapest

R.S. Moreland, Philadelphia, PA S. Moreland, Princeton, NJ

A. Nasjletti, Valhalla, NY J. Navas, Atlanta, GA T.O. Neild, Clayton R.M. Nerem, Ecublens H. Nielsen, Aarhus H. Nilsson, Aarhus N.C.B. Nyborg, Aarhus H. Nygaard, Aarhus

M. O’Donnell, Davis, CA

T. Ohhashi, Matsumoto

J.D. Ollerenshaw, Atlanta, GA

G. Osol, Burlington, VT

G. Owens, CharlottesviUe, VA

R.M. Palmer, Beckenham

P.K.T. Pang, Edmonton

A.A. Parsons, Welwyn

S. Parthasarathy, La Jolla, CA

P. Pauletto, Padova

M.J. Peach, CharlottesviUe, VA $†$

J.D. Pearson, London

K. Perktold, Graz

J.B. Philips III, Birmingham, AL J.S. Pober, New Haven, CT L. Poston, London R.L. Prewitt, Norfolk, VA

U. Quast, Basel

C. Rembold, CharlottesviUe, VA R. Richter, Berlin R. Rubio, CharlottesviUe, VA N.J. Rusch, Milwaukee, WI

I. Sarembock, CharlottesviUe, VA

M. Schachter, London 
V. Schini, Houston, TX

T. Scott-Burden, Houston, TX

C.L. Seidel, Houston, TX

W. Sessa, CharlottesviUe, VA

R. Simon, Kiel

H.A. Singer, Danville, PA

K. Skajaa, Aarhus

L.L. Slakey, Amherst, MA

A.V. Somlyo, CharlottesviUe, VA

S.S. Sørensen, Aarhus

J.R. Spears, Detroit, MI

D.R. Spitz, CharlottesviUe, VA

N.C. Spurway, Glasgow

N.B. Standen, Leicester

J.C. Stoclet, Illkirch

J.D. Swales, Leicester

K. Thestrup-Pedersen, Aarhus

$\mathrm{T}$. Thien, Nijmegen

S. Thom, London

B. Thurston, Leicester

N. Toda, Ohtsu

J. Tooke, Exeter

W.R. Tracey, Abbott Park, IL

C.R. Triggle, Calgary

P. Vallance, London

E. van Bavel, Amsterdam

D. Van Riper, CharlottesviUe, VA

R.M. Wadsworth, Glasgow J.A. Waldhausen, Hershey, PA M.G Walker, Manchester P.M. Walker, Toronto D.J. Webb, Edinburgh R.C. Webb, Ann Arbor, MI P. Weissberg, Cambridge N. Westerhof, Amsterdam R.J. Winquist, Ridgefield, CT M.S. Wolin, Valhalla, NY S. Wray, Liverpool

K. Yoshida, Ohtsu

A. Zembowicz, London 
Thanks to the Reviewers 